
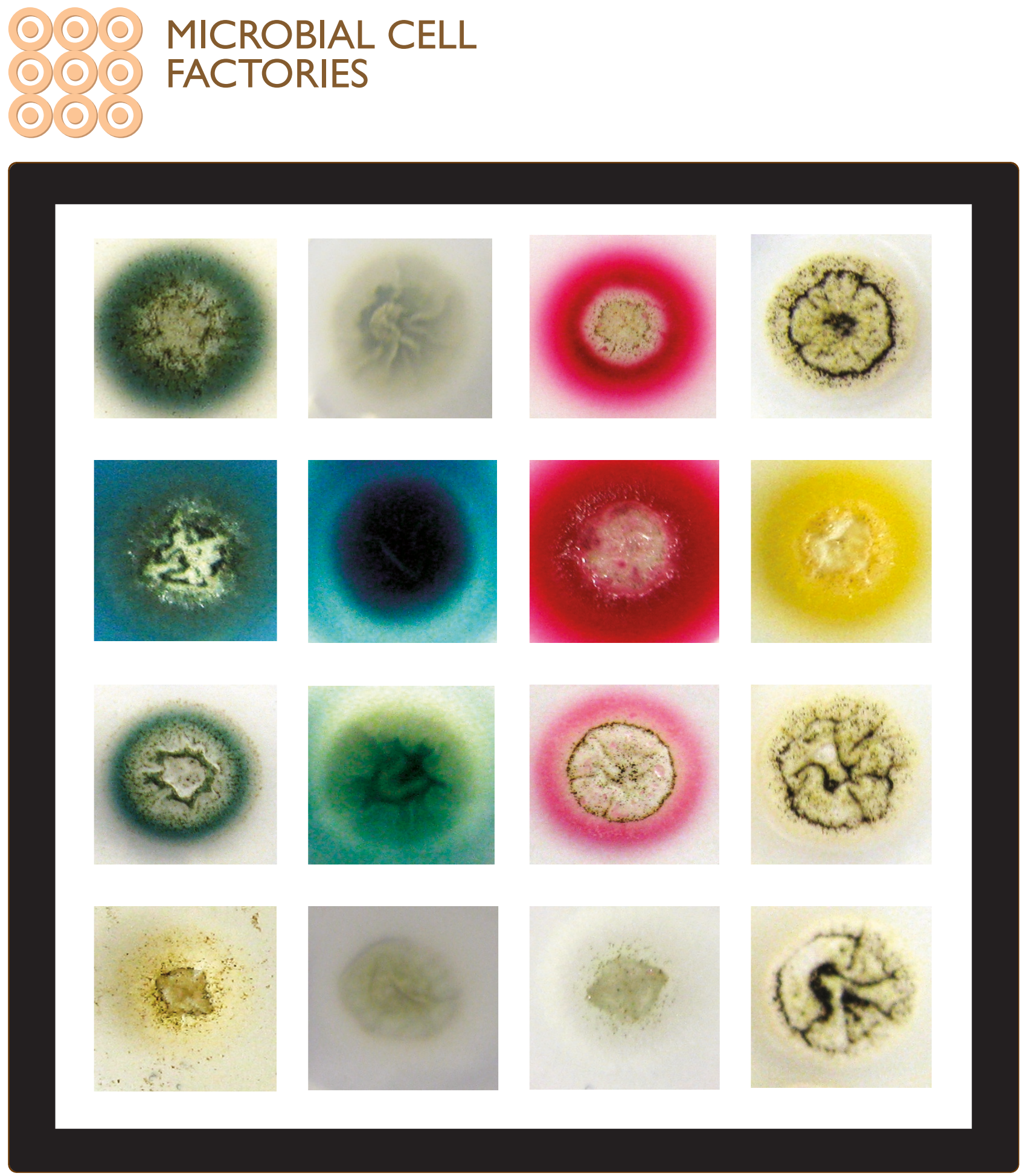

The Aspergillus niger multicopper oxidase family: analysis and overexpression of laccase-like encoding genes

Tamayo Ramos et al.

C Biomed Central 


\title{
The Aspergillus niger multicopper oxidase family: analysis and overexpression of laccase-like encoding genes
}

\author{
Juan A Tamayo Ramos', Sharief Barends ${ }^{2,3}$, Raymond MD Verhaert ${ }^{2}$ and Leo H de Graaff ${ }^{*}$
}

\begin{abstract}
Background: Many filamentous fungal genomes contain complex groups of multicopper oxidase (MCO) coding genes that makes them a good source for new laccases with potential biotechnological interest. A bioinformatics analysis of the Aspergillus niger ATCC 1015 genome resulted in the identification of thirteen MCO genes. Ten of them were cloned and homologously overexpressed.

Results: A bioinformatic analysis of the A. niger ATCC 1015 genome revealed the presence of 13 MCO genes belonging to three different subfamilies on the basis of their phylogenetic relationships: ascomycete laccases, fungal pigment MCOs and fungal ferroxidases. According to in silico amino acid sequence analysis, the putative genes encoding for functional extracellular laccases (mcoA, mcoB, mcoC, mcoD, mcoE, mcoF, mcoG, mcol, mcoJ and mcoM) were placed under the control of the glaA promoter and overexpressed in A. niger N593. Enzyme activity plate assays with several common laccase substrates showed that all genes are actually expressed and code for active MCOs. Interestingly, expressed enzymes show different substrate specificities. In addition, optimization of fungal pigment MCOs extracellular production was investigated. The performance of the widely used glucoamylase signal sequence (ssGlaA) in McoA secretion was studied. Results obtained suggest that ssGlaA do not yield higher levels of secreted McoA when compared to its native secretion signal. Also, McoB synthesis was investigated using different nitrogen sources in minimal medium liquid cultures. Higher yields of extracellular McoB were achieved with $\left(\mathrm{NH}_{4}\right)_{2}$ tartrate.

Conclusions: Aspergillus niger is a good source of new laccases. The different substrate specificity observed in plate assays makes them interesting to be purified and biochemically compared. The homologous signal sequence of McoA has been shown to be a good choice for its extracellular overexpression. From the nitrogen sources tested $\left(\mathrm{NH}_{4}\right)_{2}$ tartrate has been found to be the most appropriate for McoB production in A. niger.
\end{abstract}

Keywords: Multicopper oxidase, laccase, Aspergillus niger, secretion

\section{Background}

Multicopper oxidases (MCOs) form a family of enzymes that is widely distributed in nature, including laccases (EC 1.10.3.2), ascorbate oxidases (EC 1.10.3.3), bilirubin oxidases (EC 1.3.3.5) and ferroxidases (EC 1.16.3.1) $[1,2]$. MCOs catalyze the oxidation of a variety of substrates (mainly aromatic compounds and metals) concomitantly with the reduction of molecular oxygen to

\footnotetext{
* Correspondence: leo.degraaff@wur.nl

${ }^{1}$ Fungal Systems Biology, Laboratory of Systems and Synthetic Biology, Wageningen University, Dreijenplein 10, 6703 HB, Wageningen, The Netherlands

Full list of author information is available at the end of the article
}

water. Their ability to catalyze reactions by producing just water as the only by-product has increased the interest of the industry for their use as 'green' catalysts, and their importance is reflected in the broad spectrum of applications, that range from pulp delignification, textile dye bleaching and water or soil detoxification, to the formation of pigments, the development of clinical tests and applications in the field of biosensors, bioreactors, and biofuel cells $[2,3]$. Laccases form the biggest subgroup within the MCO family, and due to their broad substrate specificity they attract the most attention in studies for biotechnological applications, although other

\section{Biomed Central}


family members like ascorbate oxidases and bilirubin oxidases have shown to be promising as biocatalysts for the industry as well $[2,3]$. Fungal pigment MCOs are able to oxidize typical laccase substrates as $p$-phenylenediamines, pyrogallol, gallic acid or 2,2-azino-di(3-ethylbenzthiazoline) sulfonic acid (ABTS) $[4,5]$, but no studies about their possible industrial applications have been reported.

MCO coding genes appear to be redundant in fungal genomes, probably due to the fact that their coding products may play different physiological roles and they can be differently regulated depending on environmental conditions $[3,6]$. This phenomenon has been described mostly in basidiomycetes like Coprinopsis cinerea, that contains 17 laccase sensu stricto coding genes [7] and Laccaria bicolor with 9 laccase sensu stricto and 2 ferroxidase genes [8]. In ascomycetes MCOs have been much less studied [9]. Aspergillus nidulans produces YA [10] and LccD [5], belonging to the fungal pigment MCO subgroup and the laccases sensu stricto LccB and LccC [5]. Distinct MCO genes and their coding products have been also characterized in Neurospora crassa, Aspergillus fumigatus, Myceliophthora thermophila, Melanocarpus albomyces, and more recently in Trichoderma reesei [11-15]. The production of active laccases has been reported in many other ascomycetes [16], but no complete families in fungi from this phylum have been described so far. Therefore, it is difficult to have an actual overview about the biotechnological potential of ascomycete genomes as source for new MCOs. Regarding Aspergillus niger, widely used as an amenable tool for laccase production together with other cell factories like Pichia pastoris, T. reesei or Aspergillus oryzae [17-19], it is remarkable that limited information is available about its potential as multicopper oxidases source, whereas various oxidoreductases, including a laccase from black aspergilli, are already being industrially commercialized (http://www.amfep.org/documents/ Amfep List of Commercial Enzymes - OCT2009 Amfep 09 68.pdf). Only recently, BrnA, a MCO involved in the conidial pigmentation of $A$. niger has been described [20]. The fact that a large number of threedimensional structures of basidiomycete laccases have been published, whereas only two 3D structures from the ascomycete laccases sensu stricto of Melanocarpus albomyces [21] and Thielavia arenaria [9] have been reported, and no one is available from the fungal pigment MCO subfamily, also indicates the lack of information generated from ascomycete MCOs, particularly from the latter group, when compared to basidiomycetes.

In this investigation ten $A$. niger $\mathrm{MCO}$ putative genes were selected on the basis of their phylogenetic relationships with other multicopper oxidases with reported laccase activity and homologously expressed from the glaA promoter. Optimization of the extracellular levels of fungal pigment MCOs was investigated using different approaches.

\section{Results and discussion}

Identification and phylogenetic characterization of $A$. niger MCOs

A BLASTp search was made against the $A$. niger ATCC 1015 genome to identify its complete MCO coding gene family. The sequences of 38 characterized multicopper oxidases, from different origin and with different biological roles, were used as query. As a result, 13 gene models containing the residues proposed to be involved in copper coordination (Figure 1) -a characteristic signature of all MCOs- were identified (Table 1), including the recently characterized BrnA [20]. Sequence identities among the 13 deduced proteins ranged from 21 to $69 \%$ with McoE and McoI having the lowest identity to the others $(35 \% \leq)$. McoA, McoB and McoC where at least $52 \%$ identical among them, whereas McoD, McoF and McoG were no less than $50 \%$ identical between themselves. In addition $\mathrm{McoH}$ and McoK showed the highest degree of identity (69\%). Signal P 3.0 predicted a signal peptide for all the deduced proteins with the exception of McoI, suggesting therefore an intracellular localization for the latter. However the SecretomeP 2.0 Server, a tool designed to predict whether a protein is secreted via non-classical pathways that has been used repeatedly in the analysis of fungal sequences [22-25], predicted that McoI might be an extracellular protein. The TMHMM Server 2.0 predicted a transmembrane domain in the C-terminal region of $\mathrm{McoH}$ and McoK.

In order to identify those $A$. niger MCOs that are more likely to have laccase activity, a phylogenetic analysis was performed to investigate their relationships with a number of already characterized multicopper oxidases from different origins (Figure 2).

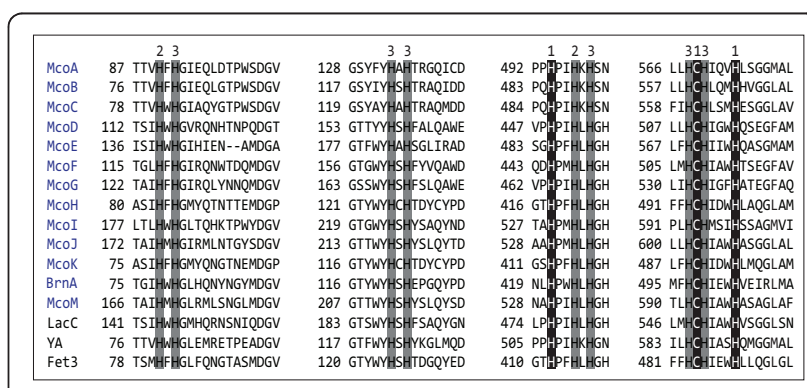

Figure 1 Sequence alignment of the four copper-binding sites in the 13 A. niger ATCC 1015 MCOs with N. crassa ascomycete laccase (LaCC), S. cerevisiae ferroxidase (Fet3) and A. nidulans fungal pigment MCO (YA). Numbers indicate the histidine $(H)$ and cysteine (C) copper ligands, according to the type of $\mathrm{Cu}$ they bind [56]. 
Table 1 Protein accession number, predicted nucleotide and amino acid sequence length of the A. niger ATCC 1015 and CBS 513.88 strains MCOs

\begin{tabular}{|c|c|c|c|c|}
\hline Gene & Accession $n^{\circ}$ ATCC 1015 & Accession $n^{\circ}$ CBS 513.88 & Nucleotide sequence length (bp) & Deduced primary polypeptide length (aa) \\
\hline$m c O A$ & e_gw1_1.1387 & An01g14010 & 1907 & 602 \\
\hline$\overline{m c o B}$ & e_gw1_1.1368 & An01g13660 & 1922 & 596 \\
\hline mcoC & fge1_pg_C_14000044 & An03g03750 & 1979 & 614 \\
\hline$m c o D$ & e_gw1_4.1637 & An11g03580 & 1806 & 563 \\
\hline mCOE & fge1_pg_C_1000314 & An01g11120 & 2143 & 586 \\
\hline mcoF & fge1 e_gw1_12.409 & An05g02340 & 1876 & 559 \\
\hline mcoG & fge1_pm_C_3000179 & An08g08450 & 1840 & 594 \\
\hline $\mathrm{mcOH}$ & e_gw1_1.309 & An01g08960 & 1842 & 613 \\
\hline mcol & gw1_10.607 & An18g02690 & 2188 & 648 \\
\hline mcol & gw1_9.210 & An12g05810 & 2491 & 672 \\
\hline mcoK & fge1_pg_C_6000422 & An15g05520 & 1845 & 614 \\
\hline brnA & e_gw1_8.661 & An14g05370 & 1722 & 547 \\
\hline mcoM & gw1_7.235 & An16g02020 & 2413 & 658 \\
\hline mcoN & - & An01g00860 & 1856 & 598 \\
\hline mcoO & - & An04g10400 & 1946 & 605 \\
\hline mCOP & - & An05g02540 & 1903 & 588 \\
\hline
\end{tabular}

The products of genes $m c o A, m c o B$ and $m c o C$ cluster with fungal pigment MCOs. This group contains the enzymes YA from A. nidulans and Abr2p from A. fumigatus, both involved in conidial pigment biosynthesis and reported to have laccase activity, and TilA, which is localized at the growing hyphal tip in A. nidulans and has unknown function $[10,15,26]$.

McoD, McoF, McoG, McoI, McoJ and McoM are in the cluster that groups the ascomycetous laccases (sensu stricto) that contains characterized laccases from Botrytis cinerea [27], N. crassa [14], M. albomyces [13] and A. nidulans [5] among others.

The MCOs McoH, McoK and BrnA cluster in the fungal ferroxidase family, that includes the Fet3 and Fet5 proteins, responsible for the reductive iron assimilation system in Saccharomyces cerevisiae [28,29]. This cluster also harbors the vermelone dehydratase Abr1, involved (together with Abr2) in the biosynthetic pathway of melanin in A. fumigatus [30,31]. McoH and McoK are both $49 \%$ identical to Fet3 and their coding genes are clustered with putative iron permease genes (ftrA and $f \operatorname{tr} B$ ) whose coding products are $51 \%$ and $48 \%$ identical to S. cerevisiae Ftr1 respectively. Fet3 and Ftr1 are responsible of the high affinity iron transport in $S$. cerevisiae, therefore a similar function is predicted for $\mathrm{McoH}$ and $\mathrm{McoK}$ in $A$. niger.

McoE belongs to the ferroxidases/laccases subgroup, a grade of basidiomycete and ascomycete MCOs that includes Mco1 from Phanerochaete chrysosporium and a laccase from Cryptococcus neoformans, both of them with strong ferroxidase and weak laccase activities [1].
The number of $\mathrm{MCO}$ coding genes present in $A$. niger ATCC 1015 is the highest reported so far in any ascomycete, and higher than those reported in basidiomycetes, with the exception of Coprinopsis cinerea, whose genome contains $17 \mathrm{MCO}$ coding genes [7,8]. Also, significant genotypic differences have been recently found between $A$. niger strains [32]; the genome mining of the CBS 513.88 strain revealed the presence of $16 \mathrm{MCO}$ genes (Table 1). Of these, 13 homologous to the ones present in ATCC 1015 and three additional full-length coding genes: the ascomycete laccase $\mathrm{McoN}$, similar to McoI (58\% identical) and the fungal pigment MCOs $\mathrm{McoO}$ and McoP, similar to McoA (56\% identical) and McoC (57\% identitical) respectively, highlighting even more the potential of $A$. niger as an interesting source for new multicopper oxidases.

\section{Homologous overexpression of $A$. niger MCO coding genes with possible biotechnological interest}

The analysis of the amino acid sequences and the phylogenetic relationships of the putative A. niger ATCC 1015 MCOs indicates that at least 9 of the 13 coding genes could code for extracellular enzymes with laccase activity. As mentioned before, laccases have been reported both in fungal pigment $\mathrm{MCO}$ and ascomycete laccase sensu stricto subgroups, thus all $A$. niger $\mathrm{MCO}$ genes included in the two subfamilies and predicted to be extracellular were selected for their homologous overexpression. The $m c o I$ gene was also selected as it could be targeted to a non-classical secretory pathway and its hypothetical coding product is $49 \%$ identical to 


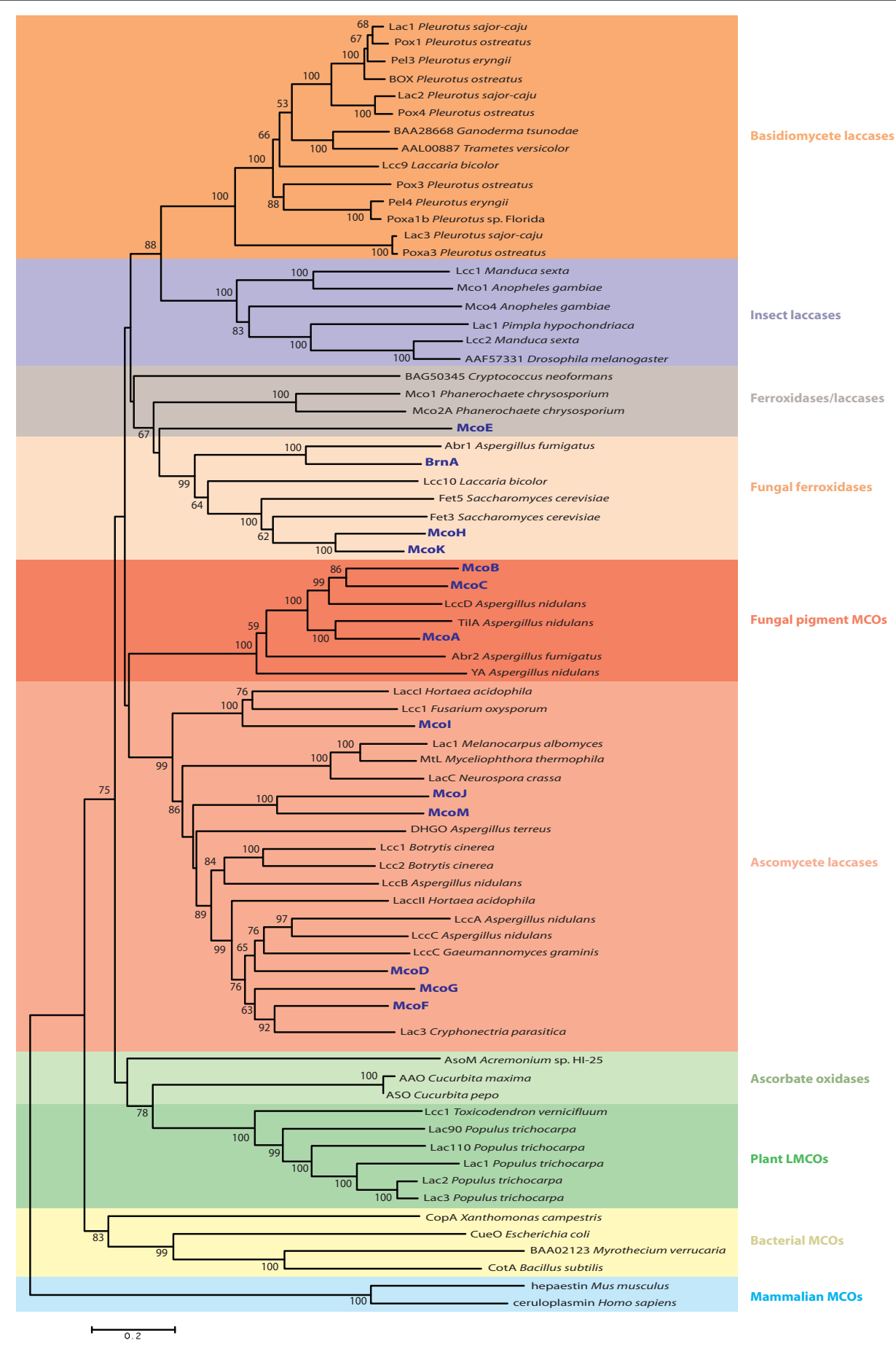

Figure 2 Neighbor joining tree of multicopper oxidase amino acid sequences. The A. niger MCOs (in dark blue) are distributed amongst the ascomycete laccases, fungal pigment MCOs, fungal ferroxidases and ferroxidases/laccases subfamilies. The phylogenetic tree was constructed with MEGA4, using the Poisson correction model, pairwise deletion and 500 replicates. Only values $\geq 50 \%$ are shown.

the laccase I of Hortaea acidophila [33]. In addition $m c o E$ from the ferroxidases/laccases grade was selected, as its coding product is predicted to be an extracellular enzyme and no similar MCO has been characterized so far. $\mathrm{McoH}$ and $\mathrm{McoK}$ have been predicted to be iron transporters located in the plasma membrane, therefore both of them were discarded, whereas BrnA was not selected, as no laccase activity has been reported for it and its ortolog Abr1 in $A$. fumigatus [20,30,31]. 
$m c o A-G, m c o I, m c o J$ and $m c o M$ were homologously expressed in the $A$. niger N593 strain under the control of the widely used glaA-promoter $\left(g l a A_{\mathrm{p}}\right)$ [34]. Due to probable ectopic integrations of the plasmids carrying the $\mathrm{MCO}$ genes, different expression efficiencies can occur. Therefore at least 15 transformants expressing each gene were tested in plate activity assays for ABTS and 4-amino-2,6-dibromophenol/3,5-dimethylaniline (ADBP/DMA) conversion. A significant number of transformants per gene showed laccase activity against one or both substrates. As expected, differences were observed in expression levels for each group of transformants. The additional file 1 in the supplementary material illustrates the screening for McoJ positive transformants (around 60\%) in an ADBP/DMA plate assay. Different expression levels can be observed: from no detectable activity to a maximum rate of ADBP/ DMA conversion, reached by several transformants.

When ADBP/DMA was used as a substrate laccase activity was detected in a significant number of transformants expressing McoA, McoB, McoC, McoD, McoF, McoG and McoJ. Only strains expressing McoB, McoC, McoD, McoG and McoJ showed laccase activity towards ABTS. Clear differences in substrate specificity were also reported for A. nidulans laccases when ADBP/ DMA and ABTS were used in plate assays [5]. Therefore, in order to have more insight in A. niger MCOs substrate specificity two more laccase substrates - N, Ndimethyl- $p$-phenylenediamine sulfate (DMPPDA) and 2,6-dimethoxyphenol (DMP) - were tested in plate assays. Again, at least 15 transformants expressing McoE, McoI and McoM were tested, whereas for the other MCOs (McoA, McoB, McoC, McoD, McoF, McoG and McoJ), only the transformant with the higher activity towards the substrates used previously was tested in parallel. For these substrates also different patterns of enzyme activity were observed: only McoA, McoB, McoC, McoD and McoG reacted with DMPPDA, whereas McoB, McoE, McoF, McoG, McoI and McoM transformants showed activity when assayed with DMP. Different degrees of specificity could be observed too depending on the color intensity of the reaction product: green-blue halo for ADBP/DMA, green halo with ABTS oxidation, pink halo for DMPPDA and yellow halo in the case of DMP (Figure 3).

The substrate specificity of $A$. niger MCOs appeared not to be correlated to their primary structures, as both ascomycete laccase sensu stricto and fungal pigment MCO subgroups include enzymes that were able to oxidize the four substrates: McoB and McoG; enzymes that clearly reacted with all of them but DMP: McoC and McoD; and enzymes that only showed activity with two substrates: McoA (ADBP/DMA and DMPPDA), McoF (ADBP/DMA and DMP) and McoJ (ADBP/DMA and

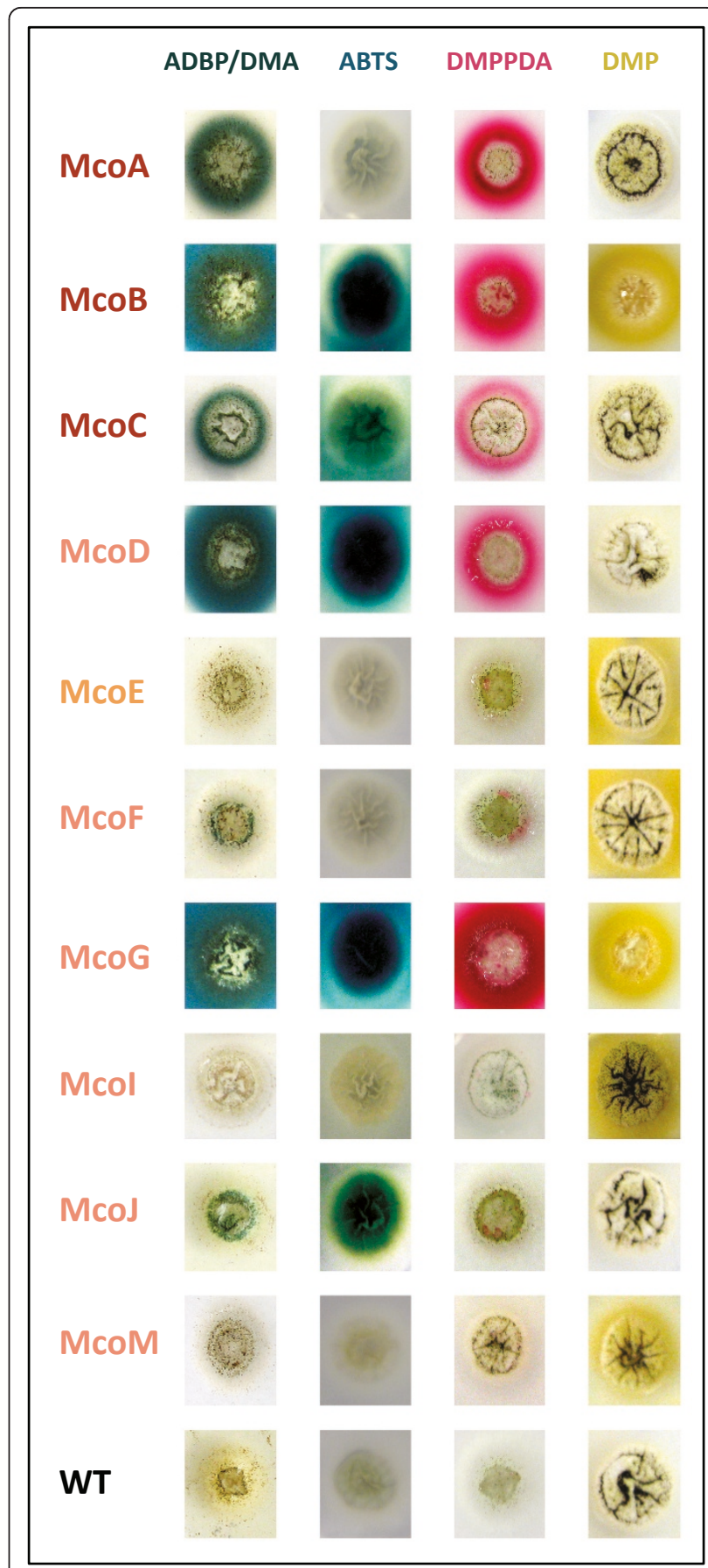

Figure 3 Enzyme activity plate assays of $A$. niger N593 strain expressing McoA, McoB, McoC, McoD, McoE, McoF, McoG, Mcol, McoJ and McoM in the presence of substrates ADBP/ DMA, ABTS, DMPPDA and DMP. WT refers to A. niger N593 transformed with the empty vector pALIV.

ABTS). McoE, McoI and McoM were only able to oxidize DMP and very weakly, as the oxidation of the substrate was only visible after four days. However, as reported for other fungal MCOs, they may need other conditions like: a different concentration of $\mathrm{CuSO}_{4}$, 
substrate, incubation temperature and/or $\mathrm{pH}$ to perform their activity optimally $[16,35]$.

The patterns of activity observed in plate assays for each A. niger MCO against the different substrates suggest that remarkable biochemical differences exist between them. Therefore, further studies should be done with respect to their biochemical characterization and their possible biotechnological potential.

\section{Optimization of fungal pigment MCOs extracellular production}

Due to the wide range of possible applications of multicopper oxidases many studies are focusing on the improvement of their production levels and their molecular characterization [36]. Fungal pigment MCOs are still a far less explored group of copper-containing polyphenol oxidases in comparison to laccases sensu stricto. Although some of them have been homologous expressed in $A$. nidulans, being used as a reporter system [5], no overproduction studies have been described. In that sense, the performance of the GlaA signal sequence (ssGlaA), successfully used to improve the secretion of many proteins, including recombinant laccases [37-39], as well as different culture conditions, were analyzed in this work.

To investigate the effect of ssGlaA in the production of extracellular fungal pigment MCOs McoA was chosen as a model enzyme. A. niger transformants ectopically expressing McoA with ssGlaA instead of their native signal sequence (ssMcoA) were generated, and their performance compared with McoA producers with ssMcoA.

Thirty transformants per construct were grown in 96 well culture plates using minimal medium with $50 \mathrm{mM}$ maltose and $0.1 \mathrm{mM} \mathrm{CuSO}_{4}$. After 24 hours McoA production was quantified using $\mathrm{ADBP} / \mathrm{DMA}$ as a substrate. Figure 4 shows the McoA activity of the sixty transformants, expressed in units $\mathrm{mg}^{-1}$ total protein secreted. The strains based on the ssMcoA resulted to produce on average higher levels of extracellular McoA in comparison to ssGlaA strains. The best producer carrying ssMcoA was able to produce $90 \%$ more enzyme than the best one with ssGlaA, whereas the difference in McoA production between the top five producers from both conditions was around $70 \%$. This result indicates that GlaA signal sequence is not more efficient than ssMcoA in the production of extracellular McoA. However, further experiments should be performed to elucidate whether ssGlaA produces a positive effect in $\mathrm{McoB}$ or McoC extracellular levels.

To investigate the effect of different culture conditions McoB was used as a reporter. As the effect of a number of carbon sources in the performance of the GlaA expression system has been recently deeply investigated

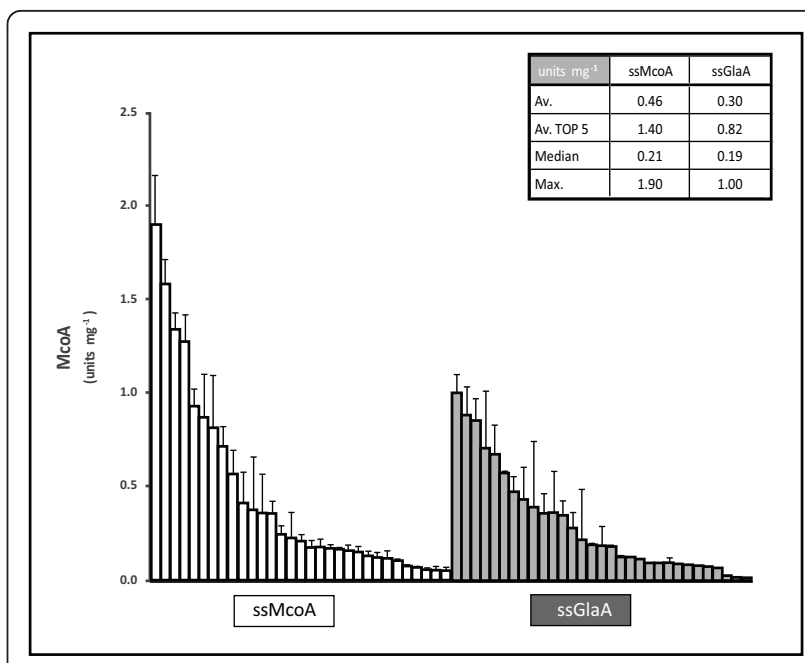

Figure 4 Laccase activity (units $\mathrm{mg}^{-1}$ total secreted protein) in culture supernatants of $A$. niger N593 transformants expressing McoA with its native secretion signal (ssMcoA) (white bars) and with ssMcoA replaced by the glucoamylase leader sequence (ssGlaA) (grey bars). The embedded table shows the average (Av.), the average of the five best producers (Av. TOP 5), the median and the higher levels (Max.) of McoA secreted by each group of transformants.

[40], various organic and inorganic nitrogen sources were tested in this work. The best McoB producer out of 15 strains tested in plate assays was grown in $100 \mathrm{~mL}$ of minimal medium with the following nitrogen sources: urea, $\mathrm{NH}_{4} \mathrm{CL}$, casamino acids (CAA), $\mathrm{NaNO}_{3}$ and $\left(\mathrm{NH}_{4}\right)$ ${ }_{2}$ tartrate. Culture supernatant samples were taken 24 and 48 hours after inoculation and McoB production was quantified using DMPPDA as a substrate. Only detectable amounts of $\mathrm{McoB}$ were measured when $\mathrm{NaNO}_{3}$ and $\left(\mathrm{NH}_{4}\right)_{2}$ tartrate were present in the media. Additionally, McoB production in cultures with $\mathrm{NaNO}_{3}$ and $\left(\mathrm{NH}_{4}\right)_{2}$ tartrate supplemented with $0.1 \%$ of CAA were also tested (Figure 5). As shown in Figure 5, cultures with $\left(\mathrm{NH}_{4}\right)_{2}$ tartrate yielded higher levels of McoB, independently of the sampling point or the absence/presence of CAA. At 24 hours after inoculation McoB activity was 8 times higher in $\left(\mathrm{NH}_{4}\right)_{2}$ tartrate cultures when no CAA were added and 13 times higher with CAA. At 48 hours McoB activity was still 2.7 and 1.8 times higher when $\left(\mathrm{NH}_{4}\right)_{2}$ tartrate was used as a substrate, in the absence or presence of CAA respectively. Therefore $\left(\mathrm{NH}_{4}\right)_{2}$ tartrate is the best choice for McoB production over all nitrogen sources and conditions tested in this study. Also, in contrast to the positive effects reported in the $A$. niger GlaA expression when the culture medium was supplemented with $0.5 \%$ CAA [41], no clear positive effect was observed in McoB production with a supplement of $0.1 \%$ of CAA. On the other hand, although no high titres of $\mathrm{McoB}$ were 


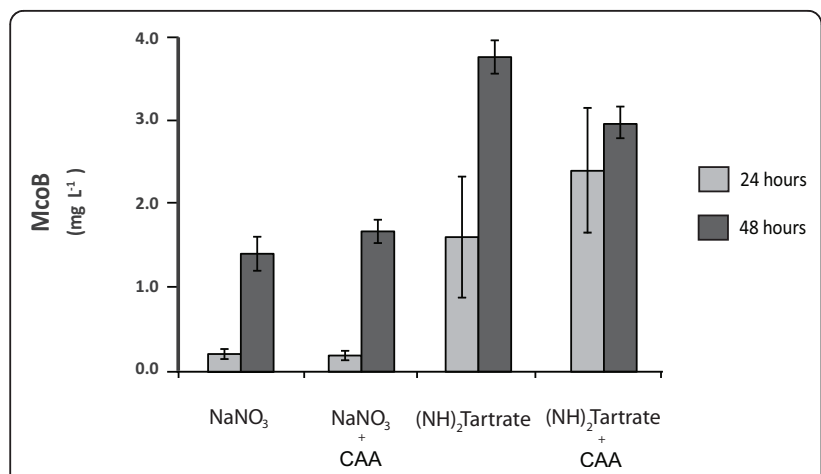

Figure $5 \mathrm{McoB}\left(\mathrm{mg} \mathrm{L}^{-1}\right)$ in supernatants of cultures containing different nitrogen sources at 24 (clear grey bars) and 48 (dark grey bars) hours after inoculation. The concentration of $\mathrm{NaNO}_{3}$ and $\left(\mathrm{NH}_{4}\right)_{2}$ tartrate was $70 \mathrm{mM}$. CAA as a supplement was used in a final concentration of $0.1 \%$.

achieved in the culture conditions studied, a further screening of transformants has allowed the isolation of strains able to produce around 10 times higher levels to the ones presented in Figure 5 (Tamayo-Ramos et al., manuscript in preparation). The use of strategies based on efforts done so far to boost yields of recombinant proteins (in particular laccases) [40-44], should result in new increases of McoB production levels.

\section{Conclusions}

The complete family of $A$. niger MCO coding genes was identified in the acidogenic wild-type strain ATCC 1015.
Those genes coding for putative extracellular laccaselike MCOs were selected after the analysis of their theoretical coding products primary structure and phylogenetic relationships. After their cloning and overexpression in A. niger different plate enzyme activity assays showed that all genes code for active multicopper oxidases, with apparent different degrees of affinity and specificity. The use of the $A$. niger GlaA secretion signal did not suppose an increase in McoA extracellular production and $\left(\mathrm{NH}_{4}\right)_{2}$ tartrate resulted to be the most appropriated nitrogen source for $\mathrm{McoB}$ synthesis.

Three additional MCO genes were found to be only present in the enzyme-producing strain CBS 513.88, confirming the significant genotypic differences between different $A$. niger strains and showing the potential of these ascomycete genomes as a source for new MCOs, that should be further investigated in order to get more insights about their possible biotechnological potential and their biological role.

\section{Methods}

\section{Strains and culture media}

Escherichia coli DH5 $\alpha$ was used for all DNA manipulations. The pyrA deficient strain A. niger N593 was selected for the PCR amplification and homologous expression of MCO coding genes. Complete medium plates were used for spores preparation [45] and minimal medium [45], containing $50 \mathrm{mM}$ of maltose as the sole carbon source and $0.1 \mathrm{mM}$ of $\mathrm{CuSO}_{4}$, was used

Table 2 Primers used in this work for the amplification of the MCO coding genes in A. niger N593 strain

\begin{tabular}{|c|c|c|}
\hline Gene & Primer & Sequence \\
\hline \multirow[t]{2}{*}{ mCOA } & mcoA-Fw & 5'-GACAACTTAATTAACCACCATGTCGCCCTTTCAATTCGGAC-3' \\
\hline & mcoA-Rv & 5'-TGTACAGCGGCCGCTCAGGAATCAGAGAGCTGGTACT-3' \\
\hline \multirow[t]{2}{*}{$m C O B$} & mcoB-Fw & 5'-GACAACTTAATTAACCACCATGAGTATATCTCAGAGCAGGC-3' \\
\hline & mcoB-Rv & 5'-TGTACAGCGGCCGC CTAGATTGGCATCACTGGCAAC-3' \\
\hline \multirow[t]{2}{*}{ mcoC } & mcoC-Fw & 5'-GACAACTTAATTAACCACCATGAAGTGGTCCCATCCCAAC-3' \\
\hline & mcoC-Rv & 5'-TGTACAGCGGCCGCCTAGTCAAAGCTAGGATGATCCA-3' \\
\hline \multirow[t]{2}{*}{ mCoD } & mcoD-Fw & 5'-GACAACTTAATTAACCACCATGCACTTGCATACTATCCTGG-3' \\
\hline & mcoD-Rv & 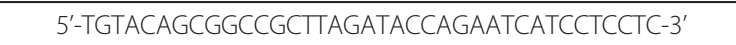 \\
\hline \multirow[t]{2}{*}{ mCOE } & mcoE-Fw & 5'-GACAACTTAATTAACCACCATGCAGCAGTCACCGTCGTTC-3' \\
\hline & mcoE-Rv & 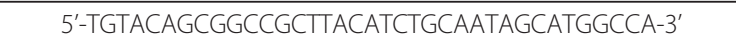 \\
\hline \multirow[t]{2}{*}{ mCoF } & mcoF-Fw & 5'-GACAACTTAATTAACCACCATGTGGTTTTCTGTCTATTTCCTT-3' \\
\hline & mcoF-Rv & 5'-TGTACAGCGGCCGC TCAAACACCCGAATCGTGCTGC-3' \\
\hline \multirow[t]{2}{*}{ mcoG } & mcoG-Fw & 5'-GACAACTTAATTAACCACCATGACAATCTITTTGCTACTCCTTGG-3' \\
\hline & mcoG-Rv & 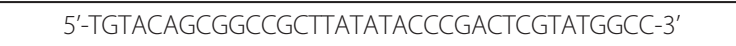 \\
\hline \multirow[t]{2}{*}{ mcol } & mcol-Fw & 5'-GACAACTTAATTAACCACCATGACTAGAACCCCACAAGTGA-3' \\
\hline & mcol-Rv & 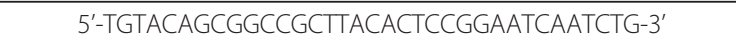 \\
\hline \multirow[t]{2}{*}{ mcol } & mcoJ-Fw & 5'-GACAACTTAATTAACCACCATGCTTTTGGAAATTTGCTGGACAG-3' \\
\hline & mcoJ-Rv & 5'-TGTACAGCGGCCGCTCAGACTCCAGAATCATCTTGGAA-3' \\
\hline \multirow[t]{2}{*}{ mCOM } & mcoM-Fw & 5'-GACAACTTAATTAACCACCATGACTCTACAAACCTACCTT-3' \\
\hline & mcoM-Rv & 5'-TGTACAGCGGCCGCTCAAATCCCCGAATCATCCTG-3' \\
\hline
\end{tabular}


both in liquid and agar plate cultures for laccase production. The concentration of all nitrogen sources in the experiment for McoB synthesis optimization was 70 mM. Casamino acids used to supplement $\mathrm{NaNO}_{3}$ and $\left(\mathrm{NH}_{4}\right)_{2}$ tartrate containing mediums were used in a final concentration of $0.1 \%$.

\section{Molecular biology techniques and transformation}

Standard methods were used for carry out DNA manipulations and E. coli transformations [46]. Genomic DNA was isolated from A. niger N593 as described previously [47]. PCR reactions were performed using proof reading Phusion polymerase (Invitrogen) according to the manufacturer's instructions. Fungal transformations were made using a similar protocol to the one described in Oliveira et al. [48].

\section{Cloning A. niger MCO genes}

Primers shown in Table 2 were used to amplify A. niger $\mathrm{MCO}$ genes. After their amplification, all genes were cloned in a pJET vector (Thermo Scientific) and their sequence verified. Plasmid pALIV (Figure 6), a derivative of pGW635 [49] carrying the promoter of the glucoamylase coding gene $\left(\right.$ gla $\left.A_{\mathrm{p}}\right)$ and the $\operatorname{trp} C$ terminator $\left(\operatorname{trp} C_{\mathrm{t}}\right)$, with $p y r A$ as a selection marker, was used as an expression vector for the $A$. niger $\mathrm{MCO}$ genes.

By means of a triple-point ligation, 234 nucleotides (nt) corresponding to the glaA 5' untranslated region (5'UTR) (synthesized by DNA 2.0 and provided in the plasmid pJref) digested with EcoRI-PacI, and the gene mcoA, obtained from the pJET vector (PacI-NotI), were ligated and placed under the control of $g l a A_{\mathrm{p}}$ and $\operatorname{trp} C_{\mathrm{t}}$ in EcoRI/ NotI digested pALIV, generating the plasmid pGWmcoA (Figure 6). Plasmids pGWmcoB, pGWmcoC, pGWmcoD, pGWmcoE, pGWmcoF, pGWmcoG, pGWmcoI, pGWmcoJ and pGWmcoM were constructed by removing $m c o A$ from pGWmcoA (PacI-NotI) and ligating the resulting linearized plasmid with PacI/NotI digested mcoB-G, $m c o I, m c o J$ and $m c o M$ respectively.

In order to compare the McoA and GlaA signal sequences, two additional plasmids, pGWssmcoA and pGWssglaA, were constructed (Figure 7). Synthetic DNA sequences coding for the first 80 residues $(240$ nucleotides) of the McoA protein, including the 23 aa of its predicted signal sequence (ssMcoA), and for 78 residues (234 nucleotides) of a fusion ssGlaA::McoA, with the glucoamylase signal sequence replacing ssMcoA, were provided by DNA 2.0 in plasmids pJssMcoA and pJssGlaA respectively. The glaA 5' UTR (202 nucleotides) was again synthesized by DNA 2.0, with different restriction sites adapted to the new cloning strategy, and provided in the plasmid pJ5ref2. Plasmid pGWmcoA was digested with EcoRI and BstEII restriction enzymes and fused in a triple point ligation with glaA 5'UTR

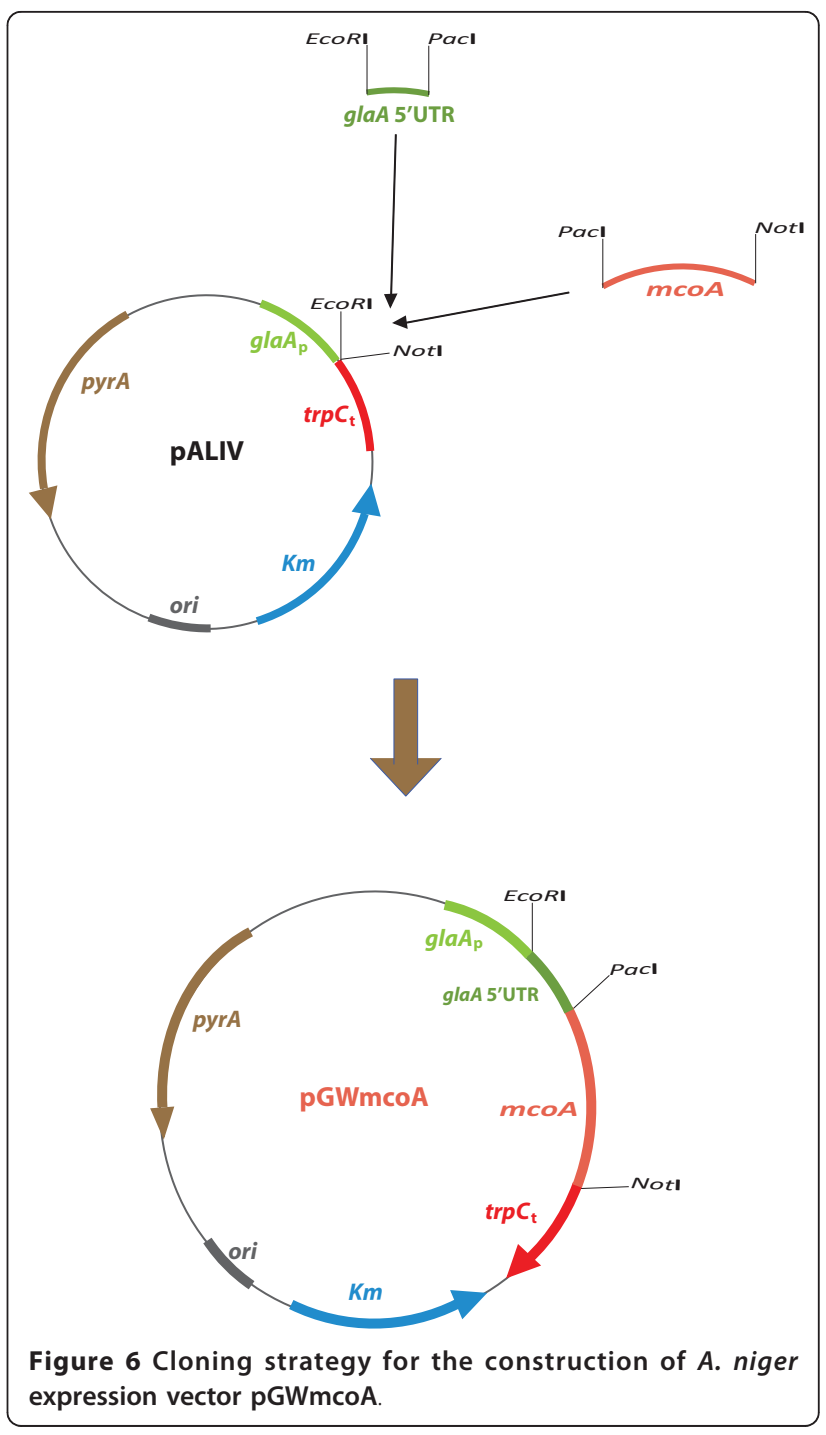

(EcoRI-PciI) and the first $240 \mathrm{nt}$ of $m c o A$ (PciI-BstEII) to yield pGWssmcoA (Figure 7a). The plasmid pGWssglaA was generated using the same procedure, through a ligation between the linearized pGWmcoA (EcoRI-BstEII), glaA 5'UTR (EcoRI-PciI) and the $234 \mathrm{nt}$ fragment coding for the ssGlaA::McoA fusion (PciI-BstEII) (Figure 7b).

\section{Enzyme activity assays}

All enzyme reactions were followed at $23^{\circ} \mathrm{C}$. All chemicals were purchased from Sigma and Invitrogen. McoA activity was quantified measuring the oxidation rate of the ADBP in a reaction mixture containing $80 \mu \mathrm{L}$ of culture supernatant and $20 \mu \mathrm{L}$ of an ADBP/DMA solution. A $5 \mathrm{mM}$ ADBP solution was prepared in $200 \mathrm{mM}$ sodium acetate buffer pH 5.0 containing $25 \%$ ethanol, in the presence of $26 \mathrm{mM}$ DMA. The product of the reaction between oxidized ADBP and DMA can be quantified by measuring its absorbance at $600 \mathrm{~nm}$. One unit of 
a)
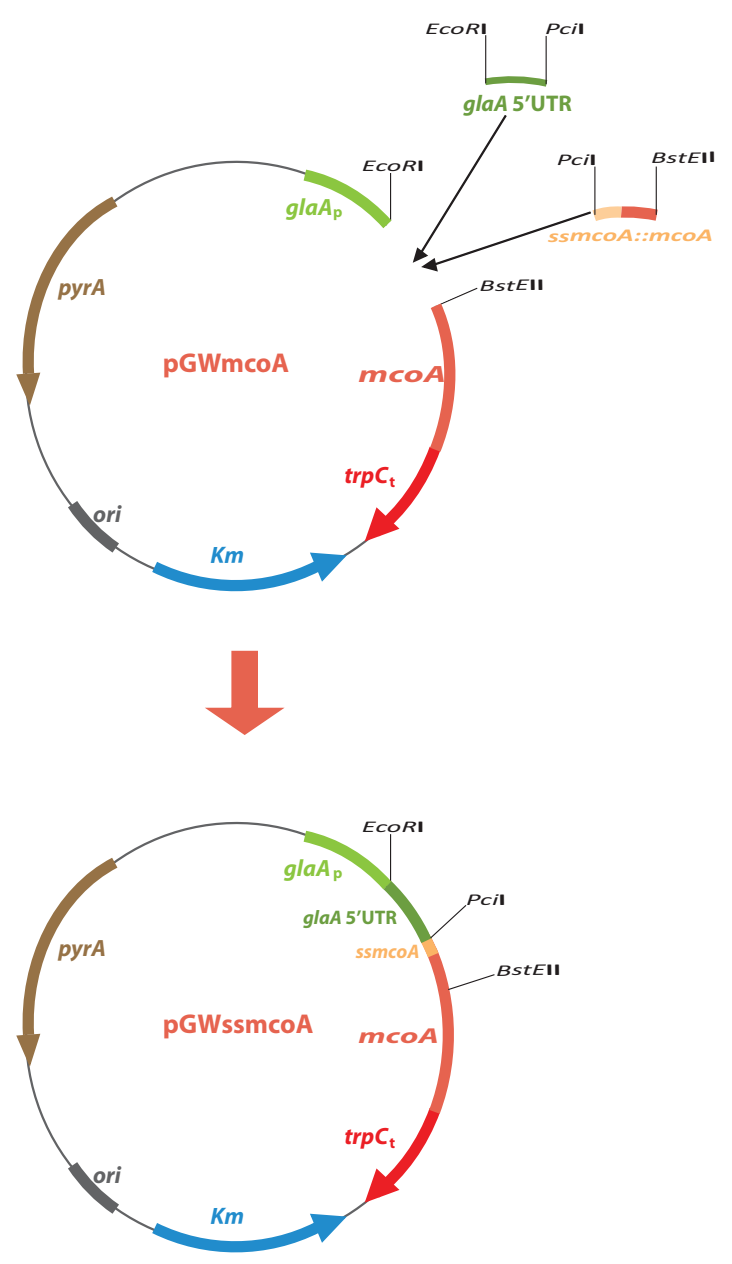

b)
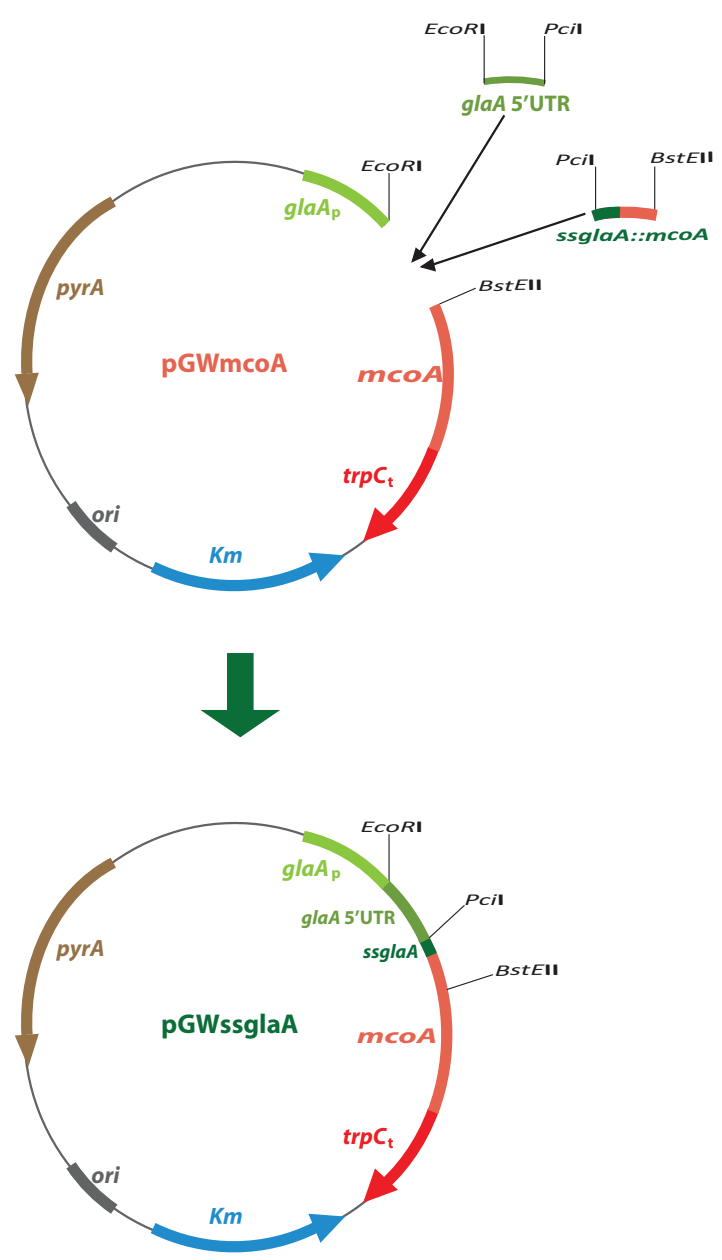

Figure 7 Cloning strategy for the construction of $A$. niger expression vectors pGWssmcoA (a) and pGWssglaA (b)

activity was defined as the change of 1.0 in optical density at $600 \mathrm{~nm}$ per minute. McoB activity was quantified in $200 \mathrm{mM}$ sodium acetate buffer $\mathrm{pH} 5.0$ measuring the oxidation rate of $10 \mathrm{mM}$ DMPPDA at $550 \mathrm{~nm}$. One unit of activity was defined as the change of 1.0 in optical density at $550 \mathrm{~nm}$ per minute. The specific activity of the enzyme was determined after the purification of the enzyme (Tamayo-Ramos et al., manuscript in preparation).

The enzyme activity assays in agar plates were performed pouring a solution of either $30 \mathrm{mM}$ DMPPDA, $5 \mathrm{mM}$ ADBP/DMA, $2 \mathrm{mM}$ DMP or $1.4 \mathrm{mM}$ of ABTS in $100 \mathrm{mM} \mathrm{NaAc}$ buffer pH 5.0 over A. niger cultures grown at $30^{\circ} \mathrm{C}$ for 48 hours. The overlay solutions were discarded after an incubation time of 5 minutes.

\section{6 well microcultures}

Microtiter plates $(1.2 \mathrm{ml}$ square-well storage plates, Thermo Scientific) containing $0.8 \mathrm{ml}$ of minimal medium were inoculated with $2 \times 10^{6}$ spores $/ \mathrm{ml}$, sealed with gas-permeable adhesive seals (Thermo Scientific) and incubated at $30^{\circ} \mathrm{C}$ and $1000 \mathrm{rpm}$ in a microtron (Infors HT). After 24 hours mycelium was pelleted by centrifugation during 15 minutes at $2000 \mathrm{~g}$ and the supernatant recovered for laccase activity measurement.

\section{Shake-fask cultures}

Erlenmeyer flasks (500 mL volume) containing $100 \mathrm{ml}$ of minimal medium were inoculated with $1 \times 10^{6}$ spores $/ \mathrm{ml}$ and incubated at $30^{\circ} \mathrm{C}$ and $250 \mathrm{rpm}$ in an orbital shaker.

\section{Bioinformatics analysis}

A. niger in silico genome mining was performed using the BLASTp tool (E value: 1e-3; Matrix: BLOSUM 62; Filter: Yes; Alignment type: gapped) in both the Aspergillus Comparative Database (strain ATCC 1015) http:// www.broadinstitute.org/ (Aspergillus Comparative 
Sequencing Project, Broad Institute of Harvard and MIT) and the Central Aspergillus Data Repository (strain CBS 513.88) http://www.cadre-genomes.org.uk/ index.html[50].

SignalP 3.0 [51] (both neural networks and Markov models methods were used), SecretomeP 2.0 (mammalian option was used) [52] and TMHMM 2.0 [53] from CBS Prediction Servers were used to predict the subcellular localization of $A$. niger MCOs. Amino acid sequences were aligned by ClustalW2 (default options provided by the program in both the pairwise alignment and the multiple sequence alignment were used) [54] from the European Bioinformatics Institute (EBI) toolbox and the phylogenetic tree was constructed with MEGA4 program [55].

\section{Additional material}

Additional file 1: ADBP/DMA plate assay screening for A. niger strains expressing $\mathbf{m c o}$ J gene. The conversion of the ADBP/DMA substrate can be observed by the formation of a green-blue color in the positive colonies. Different levels in color development indicate different McoJ activity levels. C+ refers to A. niger N593 strain expressing A. niger $\mathrm{McoB}$ as a positive control. WT refers to $A$. niger N593 transformed with the empty vector PALIV, used as a negative control.

\begin{abstract}
Acknowledgements
We thank D de Lange M Turk and K Wisse for their invaluable assistance. This work has been carried out under the IBOS SME research project 053.63.322 granted by the ACTS national research program from the Netherlands. JATR was recipient of a postdoctoral contract within research project 053.63.322.
\end{abstract}

\section{Author details}

${ }^{1}$ Fungal Systems Biology, Laboratory of Systems and Synthetic Biology, Wageningen University, Dreijenplein 10, 6703 HB, Wageningen, The Netherlands. ${ }^{2}$ ProteoNic BV, Niels Bohrweg 11-13, 2333CA Leiden, The Netherlands. ${ }^{3}$ Genencor International, 925 Page Mill Road, Palo Alto, CA 94304, USA.

\section{Authors' contributions}

JATR made the bioinformatics analysis, performed the DNA cloning and microbial transformations, designed and performed the activity plate assay experiments and the data analysis and drafted the manuscript. SB designed the cloning strategy. SB and JATR designed the microculture experiment and performed the data analysis. JATR designed and performed the shakeflask culture experiment. RV collaborated in the coordination of the research. LG designed the study and helped to draft the manuscript. All authors read and approved the submission of the manuscript.

\section{Competing interests}

The authors declare that they have no competing interests.

Received: 6 July 2011 Accepted: 8 October 2011

Published: 8 October 2011

\section{References}

1. Hoegger PJ, Kilaru S, James TY, Thacker JR, Kues U: Phylogenetic comparison and classification of laccase and related multicopper oxidase protein sequences. FEBS J 2006, 273:2308-2326.

2. Sakurai T, Kataoka K: Basic and applied features of multicopper oxidases, CueO, bilirubin oxidase, and laccase. Chem Rec 2007, 7:220-229.
3. Giardina P, Faraco V, Pezzella C, Piscitelli A, Vanhulle S, Sannia G: Laccases: a never-ending story. Cell Mol Life Sci 2010, 67:369-385.

4. Clutterbuck AJ: Absence of laccase from yellow-spored mutants of Aspergillus nidulans. J Gen Microbiol 1972, 70:423-435.

5. Mander GJ, Wang H, Bodie E, Wagner J, Vienken K, Vinuesa C, Foster C, Leeder AC, Allen G, Hamill V, et al: Use of laccase as a novel, versatile reporter system in filamentous fungi. Appl Environ Microbiol 2006, 72:5020-5026.

6. Canero DC, Roncero Ml: Functional analyses of laccase genes from Fusarium oxysporum. Phytopathology 2008, 98:509-518.

7. Kilaru S, Hoegger PJ, Kues U: The laccase multi-gene family in Coprinopsis cinerea has seventeen different members that divide into two distinct subfamilies. Curr Genet 2006, 50:45-60.

8. Courty PE, Hoegger PJ, Kilaru S, Kohler A, Buee M, Garbaye J, Martin F, Kues U: Phylogenetic analysis, genomic organization, and expression analysis of multi-copper oxidases in the ectomycorrhizal basidiomycete Laccaria bicolor. New Phytol 2009, 182:736-750.

9. Kallio JP, Gasparetti C, Andberg M, Boer H, Koivula A, Kruus K, Rouvinen J, Hakulinen N: Crystal structure of an ascomycete fungal laccase from Thielavia arenaria: Common structural features of asco-laccases. FEBS J 2011.

10. Aramayo R, Timberlake WE: Sequence and molecular structure of the Aspergillus nidulans yA (laccase I) gene. Nucleic Acids Res 1990, 18:3415

11. Berka RM, Schneider P, Golightly EJ, Brown SH, Madden M, Brown KM, Halkier T, Mondorf K, Xu F: Characterization of the gene encoding an extracellular laccase of Myceliophthora thermophila and analysis of the recombinant enzyme expressed in Aspergillus oryzae. Appl Environ Microbiol 1997, 63:3151-3157.

12. Levasseur A, Saloheimo M, Navarro D, Andberg M, Pontarotti P, Kruus K, Record E: Exploring laccase-like multicopper oxidase genes from the ascomycete Trichoderma reesei: a functional, phylogenetic and evolutionary study. BMC Biochem 2010, 11:32.

13. Kiiskinen LL, Saloheimo M: Molecular cloning and expression in Saccharomyces cerevisiae of a laccase gene from the ascomycete Melanocarpus albomyces. Appl Environ Microbiol 2004, 70:137-144.

14. Schilling B, Linden RM, Kupper U, Lerch K: Expression of Neurospora crassa laccase under the control of the copper-inducible metallothioneinpromoter. Curr Genet 1992, 22:197-203.

15. Sugareva V, Hartl A, Brock M, Hubner K, Rohde M, Heinekamp T, Brakhage AA: Characterisation of the laccase-encoding gene abr2 of the dihydroxynaphthalene-like melanin gene cluster of Aspergillus fumigatus. Arch Microbiol 2006, 186:345-355.

16. Baldrian P: Fungal laccases - occurrence and properties. FEMS Microbiol Rev 2006, 30:215-242.

17. Colao MC, Lupino S, Garzillo AM, Buonocore V, Ruzzi M: Heterologous expression of Iccl gene from Trametes trogii in Pichia pastoris and characterization of the recombinant enzyme. Microb Cell Fact 2006, 5:31.

18. Rodgers CJ, Blanford CF, Giddens SR, Skamnioti P, Armstrong FA, Gurr SJ: Designer laccases: a vogue for high-potential fungal enzymes? Trends Biotechnol 2010, 28:63-72.

19. Kunamneni A, Camarero S, Garcia-Burgos C, Plou FJ, Ballesteros A, Alcalde M: Engineering and Applications of fungal laccases for organic synthesis. Microb Cell Fact 2008, 7:32.

20. Jorgensen TR, Park J, Arentshorst M, van Welzen AM, Lamers G, Vankuyk PA Damveld RA, van den Hondel CA, Nielsen KF, Frisvad JC, Ram AF: The molecular and genetic basis of conidial pigmentation in Aspergillus niger. Fungal Genet Biol 2011, 48:544-553.

21. Hakulinen N, Kiiskinen LL, Kruus K, Saloheimo M, Paananen A, Koivula A, Rouvinen J: Crystal structure of a laccase from Melanocarpus albomyces with an intact trinuclear copper site. Nat Struct Biol 2002, 9:601-605.

22. Medina ML, Haynes PA, Breci L, Francisco WA: Analysis of secreted proteins from Aspergillus flavus. Proteomics 2005, 5:3153-3161.

23. Jami MS, Garcia-Estrada C, Barreiro C, Cuadrado AA, Salehi-Najafabadi Z, Martin JF: The Penicillium chrysogenum extracellular proteome. Conversion from a food-rotting strain to a versatile cell factory for white biotechnology. Mol Cell Proteomics 2010, 9:2729-2744.

24. Krijger JJ, Horbach R, Behr M, Schweizer P, Deising HB, Wirsel SG: The yeast signal sequence trap identifies secreted proteins of the hemibiotrophic corn pathogen Colletotrichum graminicola. Mol Plant Microbe Interact 2008, 21:1325-1336. 
25. Choi J, Park J, Kim D, Jung K, Kang S, Lee YH: Fungal secretome database: integrated platform for annotation of fungal secretomes. BMC Genomics 2010, 11:105.

26. Scherer M, Fischer R: Molecular characterization of a blue-copper laccase, TILA, of Aspergillus nidulans. FEMS Microbiol Lett 2001, 199:207-213.

27. Schouten A, Wagemakers L, Stefanato FL, van der Kaaij RM, van Kan JA: Resveratrol acts as a natural profungicide and induces self-intoxication by a specific laccase. Mol Microbiol 2002, 43:883-894.

28. Askwith C, Eide D, Van Ho A, Bernard PS, Li L, Davis-Kaplan S, Sipe DM, Kaplan J: The FET3 gene of $S$. cerevisiae encodes a multicopper oxidase required for ferrous iron uptake. Cell 1994, 76:403-410.

29. Spizzo T, Byersdorfer C, Duesterhoeft S, Eide D: The yeast FET5 gene encodes a FET3-related multicopper oxidase implicated in iron transport. Mol Gen Genet 1997, 256:547-556.

30. Tsai HF, Wheeler MH, Chang YC, Kwon-Chung KJ: A developmentally regulated gene cluster involved in conidial pigment biosynthesis in Aspergillus fumigatus. J Bacteriol 1999, 181:6469-6477.

31. Pihet $M$, Vandeputte $P$, Tronchin $G$, Renier $G$, Saulnier $P$, Georgeault $S$, Mallet R, Chabasse D, Symoens F, Bouchara JP: Melanin is an essential component for the integrity of the cell wall of Aspergillus fumigatus conidia. BMC Microbiol 2009, 9:177.

32. Andersen MR, Salazar MP, Schaap PJ, van de Vondervoort PJ, Culley D, Thykaer J, Frisvad JC, Nielsen KF, Albang R, Albermann K, et al: Comparative genomics of citric-acid-producing Aspergillus niger ATCC 1015 versus enzyme-producing CBS 513.88. Genome Res 2011, 21:885-897.

33. Tetsch L, Bend J, Holker U: Molecular and enzymatic characterisation of extra- and intracellular laccases from the acidophilic ascomycete Hortaea acidophila. Antonie Van Leeuwenhoek 2006, 90:183-194.

34. Lu X, Sun J, Nimtz M, Wissing J, Zeng AP, Rinas U: The intra- and extracellular proteome of Aspergillus niger growing on defined medium with xylose or maltose as carbon substrate. Microb Cell Fact 2010, 9:23.

35. Larrondo LF, Avila M, Salas L, Cullen D, Vicuna R: Heterologous expression of laccase cDNA from Ceriporiopsis subvermispora yields copper-activated apoprotein and complex isoform patterns. Microbiology 2003, 149:1177-1182.

36. Pezzella C, Autore F, Giardina P, Piscitelli A, Sannia G, Faraco V: The Pleurotus ostreatus laccase multi-gene family: isolation and heterologous expression of new family members. Curr Genet 2009, 55:45-57.

37. Weenink XO, Punt PJ, van den Hondel CA, Ram AF: A new method for screening and isolation of hypersecretion mutants in Aspergillus niger. Appl Microbiol Biotechnol 2006, 69:711-717.

38. Record E, Punt PJ, Chamkha M, Labat M, van Den Hondel CA, Asther M: Expression of the Pycnoporus cinnabarinus laccase gene in Aspergillus niger and characterization of the recombinant enzyme. Eur J Biochem 2002, 269:602-609.

39. Rodriguez E, Ruiz-Duenas FJ, Kooistra R, Ram A, Martinez AT, Martinez MJ: Isolation of two laccase genes from the white-rot fungus Pleurotus eryngii and heterologous expression of the pel3 encoded protein. J Biotechnol 2008, 134:9-19.

40. Ganzlin M, Rinas U: In-depth analysis of the Aspergillus niger glucoamylase $(g / a A)$ promoter performance using high-throughput screening and controlled bioreactor cultivation techniques. J Biotechnol 2008, 135:266-271.

41. Swift RJ, Karandikar A, Griffen AM, Punt PJ, van den Hondel CA, Robson GD, Trinci AP, Wiebe MG: The Effect of organic nitrogen sources on recombinant glucoamylase production by Aspergillus niger in chemostat culture. Fungal Genet Biol 2000, 31:125-133.

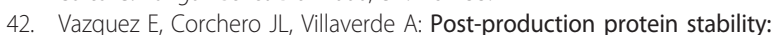
trouble beyond the cell factory. Microb Cell Fact 2011, 10:60

43. Couto SR, Toca-Herrera JL: Laccase production at reactor scale by filamentous fungi. Biotechnol Adv 2007, 25:558-569.

44. Lubertozzi D, Keasling JD: Developing Aspergillus as a host for heterologous expression. Biotechnol Adv 2009, 27:53-75.

45. van der Veen D, Oliveira JM, van den Berg WA, de Graaff LH: Analysis of variance components reveals the contribution of sample processing to transcript variation. Appl Environ Microbiol 2009, 75:2414-2422.

46. Sambrook J, Fritsch EF, Maniatis T: Molecular cloning: a laboratory manual. 2 edition. Cold Spring Harbor, N.Y.: Cold Spring Harbor Laboratory Press; 1989.

47. de Graaff $L$, van den Broek H, Visser J: Isolation and transformation of the pyruvate kinase gene of Aspergillus nidulans. Curr Genet 1988, 13:315-321.
48. Oliveira JM, van der Veen D, de Graaff LH, Qin L: Efficient cloning system for construction of gene silencing vectors in Aspergillus niger. Appl Microbiol Biotechnol 2008, 80:917-924.

49. Goosen T, van Engelenburg F, Debets F, Swart K, Bos K, van den Broek H: Tryptophan auxotrophic mutants in Aspergillus niger: inactivation of the trpC gene by cotransformation mutagenesis. Mol Gen Genet 1989, 219:282-288.

50. Mabey JE, Anderson MJ, Giles PF, Miller CJ, Attwood TK, Paton NW, Bornberg-Bauer E, Robson GD, Oliver SG, Denning DW: CADRE: the Central Aspergillus Data REpository. Nucleic Acids Res 2004, 32:D401-405.

51. Bendtsen JD, Nielsen H, von Heijne G, Brunak S: Improved prediction of signal peptides: SignalP 3.0. J Mol Biol 2004, 340:783-795.

52. Bendtsen JD, Jensen $\amalg$, Blom N, Von Heijne G, Brunak S: Feature-based prediction of non-classical and leaderless protein secretion. Protein Eng Des Sel 2004, 17:349-356.

53. Krogh A, Larsson B, von Heijne G, Sonnhammer EL: Predicting transmembrane protein topology with a hidden Markov model: application to complete genomes. J Mol Biol 2001, 305:567-580.

54. Chenna R, Sugawara H, Koike T, Lopez R, Gibson TJ, Higgins DG, Thompson JD: Multiple sequence alignment with the Clustal series of programs. Nucleic Acids Res 2003, 31:3497-3500.

55. Tamura K, Dudley J, Nei M, Kumar S: MEGA4: Molecular Evolutionary Genetics Analysis (MEGA) software version 4.0. Mol Biol Evol 2007, 24:1596-1599.

56. Morozova OV, Shumakovich GP, Gorbacheva MA, Shleev SV, Yaropolov Al: "Blue" laccases. Biochemistry (Mosc) 2007, 72:1136-1150.

doi:10.1186/1475-2859-10-78

Cite this article as: Tamayo Ramos et al:: The Aspergillus niger multicopper oxidase family: analysis and overexpression of laccase-like encoding genes. Microbial Cell Factories 2011 10:78.

\section{Submit your next manuscript to BioMed Central and take full advantage of:}

- Convenient online submission

- Thorough peer review

- No space constraints or color figure charges

- Immediate publication on acceptance

- Inclusion in PubMed, CAS, Scopus and Google Scholar

- Research which is freely available for redistribution

Submit your manuscript at www biomedcentral com/submit
C) Biomed Central 